\title{
Energy-Aware Distributed Tracking in Wireless Sensor Networks
}

\section{Abstract}

This work $[1,2]$ considers centralized distributed estimation in wireless sensor networks (WSN)

- Fusion center (FC) uses BLUE with estimate uncertainty dependent on the transmit energy and quantization levels

- Energy and bandwidth critically constrained resources in WSNs

- A convex program approximates the underlying non-convex MINLP and incorporates the node operating states into the resource allocation to prolong network lifetime

\section{Background}

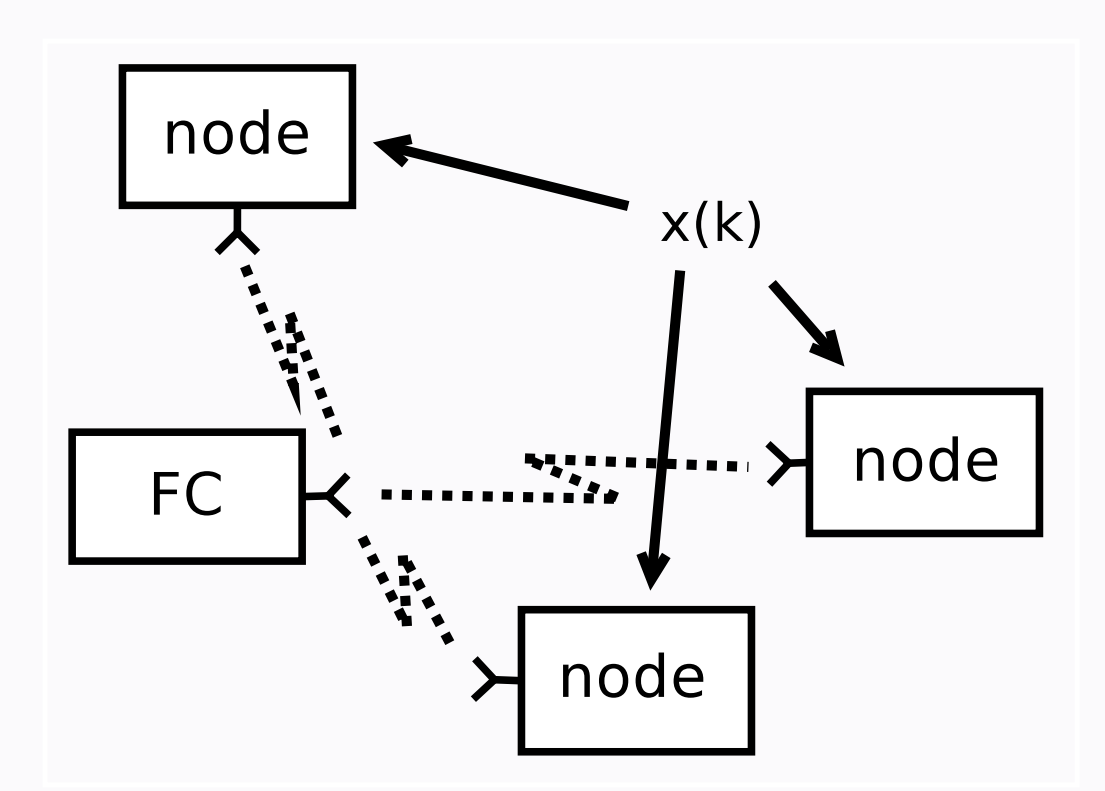

Centralized

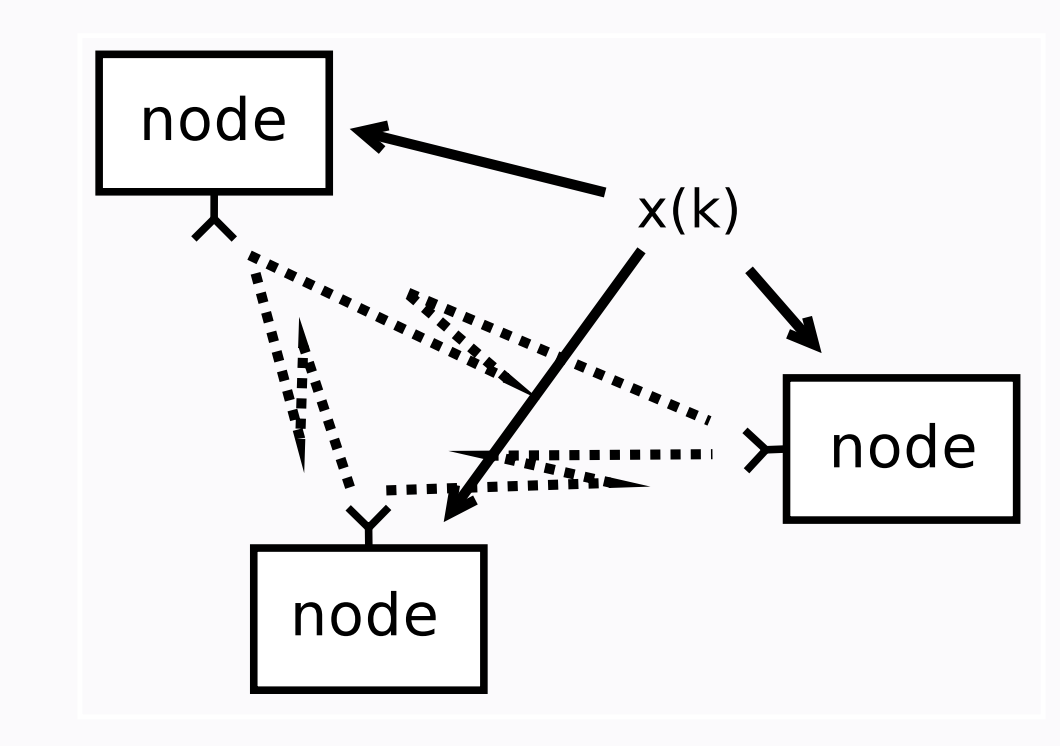

Decentralized
Advantages of Wireless Networks for Sensing

- Robust to indiv. failure, reliable, inexpensive

- Geographically distributed

- Reduce fusion node computation

Challenging Limitations

- Energy resources $\Rightarrow$ battery powered

- Transmit energy $\Rightarrow$ channel noise

- Network bandwidth $\Rightarrow$ quantization noise

\section{Prior Work}

\section{Decentralized}

Centralized-nonlinear

Measurement noise

Quantization*

BLU Estimation*

Channel, quant., \& meas. noise ${ }^{\dagger}$

Network lifetime analyzed

Distributed tracking

Balasubramanian ' $05^{\dagger}$ Williams ' $07^{\dagger}$, Varshney ${ }^{\prime} 09^{\prime}$

Channel, quant., \& meas. noise and

node operating states*

Krishnan '08

primarily consider sensor selection and scheduling, ${ }^{\ddagger}$ no communication noise considered, ${ }^{8}$ filtering is done at fusion node, ${ }^{*}$ primarily focus nestimation for a single time instance

\section{System Model}

- We consider the task of assigning bit and transmission eneroy levels after sensor selection and scheduling has been completed.

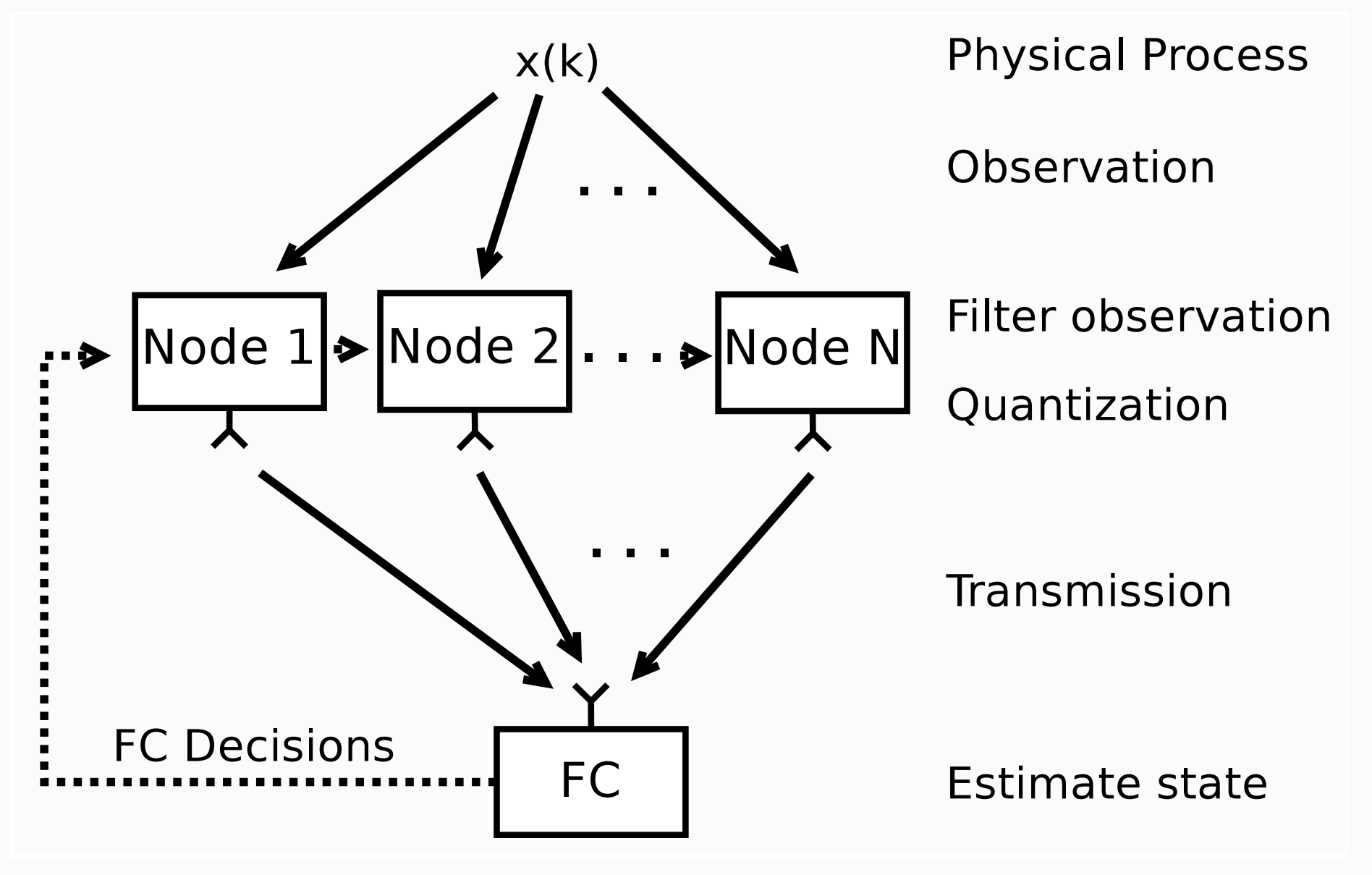

- Node estimate and covariance after the Kalman filter update $\left\{\hat{\mathbf{x}}_{n}(k \mid k), \mathbf{P}_{n}(k \mid k)\right\}$

- Received data corrupted by channel and quantization noise $\tilde{\mathbf{x}}_{n}(k \mid k)=\hat{\mathbf{x}}_{n}(k \mid k)+\mathbf{n}_{n}^{q}(k)+\mathbf{n}_{n}^{c}(k)$ - $n_{n}^{q, i}(k) \sim \mathcal{N}\left(0, r_{n}^{q, i}\right)$ and $n_{n}^{c, i}(k) \sim \mathcal{N}\left(0, r_{n}^{c, i}\right) ; \mathbf{P}_{n}^{(i, i)}(k \mid k)$ $r_{n}^{q, i}=\sigma_{q, i}^{2}(k), r_{n}^{c, i}(k)=\sigma_{c, i}^{2}$ variances of $\tilde{x}_{n}^{i}(k \mid k)$ estimate - BPSK / flat Rayleigh fading produces channel noise variance

$$
r_{n}^{c, i}(k)=\frac{4 W^{2}}{3}\left(1-\sqrt{\frac{0.5 \Gamma_{n}^{i}}{1+0.5 \Gamma_{n}^{i}}}\right)
$$

- Uniform quantization noise variance is

$$
r_{n}^{q, i}(k)=\frac{W^{2}}{3\left(2^{b_{n}^{i}(k)}-1\right)^{2}}
$$

Assumption. Make normal simplifying assumptions about noise rocesses: white, zero mean, uncorrelated; spatially and in time.

\section{Results}

Time-based single runs for scenario Single instance comparison of ap- Monte Carlo Runs prox and exact objective values - Case A: easy scenario

- Case B: energy-sensitive scenario \begin{tabular}{c|c|c|c|c|c|}
\cline { 2 - 6 } & \multicolumn{2}{c|}{ WC } & \multicolumn{2}{c|}{ LCVX } & Global \\
\cline { 2 - 6 } & \multicolumn{1}{c|}{ rlxd } & rlxd & int & \\
\cline { 2 - 6 } C.53 & 7.52 & 5.49 & 6.04 & 3.59 \\
\cline { 2 - 6 } & 11.67 & 11.21 & 9.32 & 9.21 & 5.67 \\
\hline
\end{tabular} Executed $50 \mathrm{MC}$ runs - Varied lifetime parameter $\alpha \in[0.1,1]$ : Sensitivity tests showed

Discussion of Results

- Solution is approximate, as table of objective values reveal

- Fair results, lifetime can be poor - Energy-aware heuristic improves network lifetime by $150 \%$ on average

Non-Energy-Aware

Energy-Aware

- Trade-off of estimation performance,

Error (top) \&

\section{Acknowledgements}

This work was supported in part by a Marine Corps Systems Command (MCSC) contract to M2 Technologies Inc.
Approx. \& Energy-Awareness

- Minimize $D_{i}(k)$ by minimizing $-D_{i}^{-1}(k)$

- Approximation: solve a (integer-)relaxed epigraph

form by substituting $y_{n}^{i}(k)=\mathbf{P}_{n}^{(i, i)}(k \mid k)+r_{n}^{c, i}(k)+$

$r_{n}^{q, i}(k)$ and rewriting the problem as

minimize $\sum_{i=1}^{d} \sum_{n=1}^{N} y_{n}^{i}(k)$

subject to $\quad(\mathrm{LCVX})$

$y_{n}^{i}(k)-\mathbf{P}_{n}^{(i, i)}(k \mid k)+r_{n}^{c, i}(k)+r_{n}^{q, i}(k)=0$

and still subject to constraints $\mathrm{C} 1-\mathrm{C} 5$.

- Relax the epigraph equality constraint to an inequality nstraint

$$
\mathbf{P}_{n}^{(i, i)}(k \mid k)+r_{n}^{c, i}(k)+r_{n}^{q, i}(k)-y_{n}^{i}(k) \leq 0
$$

This inequality constraint is always tight

- Use Sequential Quadratic Programming (SQP); computational cost is $O\left(\mathrm{~km}^{2}\right), k$ variables, $m$ constraints

"Worst-case" approximation

minimize $d \sum_{n=1}^{N} y^{n}(k)$

subject to

$\max _{i}\left\{\mathbf{P}_{n}^{(i, i)}(k \mid k)\right\}+r_{c}^{n}(k)+r_{q}^{n}(k)-y^{n}(k) \leq 0$

and still subject to $\mathrm{C} 1-\mathrm{C} 5$. Reduces no. of variables by $N(d-1)$ and no. of constraints by $6 N(d-1)$.

Energy-Aware Heuristic

- Dynamically update the allowable resource usage of each node based on operating state

- As a heuristic, update $(\forall n=1, \ldots, N)$

$$
\Lambda_{n}(k)=\frac{1}{\alpha+(1-\alpha) \cdot \frac{p_{n}^{r e m}(k)}{p^{i n i t}}}
$$

- Use above to replace constraint $\mathrm{C} 2$ with

$$
\Lambda_{n}(k) \cdot \sum_{i=1}^{d} p_{n}^{i}(k) b_{n}^{i}(k)<p_{n}^{r e m}(k)
$$

\section{Future Work}

- Dual problem, i.e. optmz. network lifetime

- Decentralized formulation (indep. but co-op.)

- Scheduling and selection for WSN estimation

- Effect on optimal network lifetime of adding en-

ergy harvesting systems to current model

\section{References}

References in Prior Work can be found in

. Roseveare and B. Natarajan, "Energy-Aware Distributed Trackin

N. Roseveare and B. Natarajin "Distributed Tracking

with Energy and B. Natarajan, "Distributed Tracking

Submitted: IEEE TAES, 2011 .

nload at: http: / /www-personal.k-state.edu/ nickrose 